\title{
On the use of state-observers in compartmental systems control
}

\author{
Cláudia Sousa \\ School of Education Jean Piaget \\ Piaget Institute \\ R. António Sérgio, 4410-269 \\ Canelas V.N.G., Portugal \\ Telephone: (+351) 227537600 \\ Fax: (+351) 227537681 \\ Email: cnsousa@gaia.ipiaget.org
}

\author{
Teresa Mendonça \\ Department of Applied Mathematics \\ Faculty of Sciences \\ University of Porto \\ R. do Campo Alegre, 687, 4169-007 \\ Porto, Portugal \\ Telephone: (+351) 220402202 \\ Fax: (+351) 220402209 \\ Email: tmendo@fc.up.pt
}

\author{
Paula Rocha \\ Department of Electrotecnic \\ and Computer Engineering \\ Faculty of Engineering \\ University of Porto \\ R. Dr. Roberto Frias, s/n, 4200-465 \\ Porto, Portugal \\ Telephone: (+351) 225081400 \\ Fax: (+351) 225081440 \\ Email: mprocha@fe.up.pt
}

\begin{abstract}
In this paper we analyse the performance of state-observers in the control of compartmental systems under the presence of uncertainties in the system initial state. We combine a state feedback law with positivity constraints and a state observer and prove that, as expected, the mass control objective is still attained. Moreover we show that, for a class of three-compartmental systems of interest, the resulting mass control law also allows reaching a desired steady state.

Our results are illustrated by several simulations for the control of the administration of a neuromuscular relaxant to patients undergoing surgery, which show the relevance of incorporating an observer for convergence acceleration even in case the original system is asymptotically stable.
\end{abstract}

Abstract-Index Terms-Compartmental systems, positive control, state-observers, neuromuscular blockade control.

\section{INTRODUCTION}

Compartmental systems form a subclass of positive systems that consist of a finite number of subsystems, the compartments, which exchange matter with each other and with the environment. Such systems have been successfully used to model biomedical and pharmacokinetical processes, see, for instance, [4] or [6]. Since one has to guarantee the positivity of the control input, the design of suitable control laws for such systems is more delicate. In [5], for instance, a nonnegative adaptive control law is proposed in order to guarantee the partial asymptotic setpoint stability of the closed loop system, and a positive feedback control law is proposed in [1], in order to stabilize the total system mass at an arbitrary set-point. The positive control law proposed in [1], was also used in [10] for the control of the neuromuscular blockade level (see [8], [9] and [11]) of patients undergoing surgery.

In most practical cases, the physical state of a system cannot be determined by direct observation. In these cases, we often use a state observer (see, for instance, [2] or [3]) to estimate the state from the knowledge of the input and the output of the real system. In this paper, we consider the control law referred in [1] and in [10], and analyse its performance for the target control of the total mass, when the unknown state of the system is replaced by its estimate provided by an observer. It turns out that, in this case, the asymptotic mass values coincide with those obtained when the state of the real system is supposed to be known. Moreover, we prove that for a class of three-compartmental systems of interest, the state of the controlled system also tends to the same point achieved when there is not uncertainties in the real system state. Our results are illustrated by several simulations, which, in particular, show that, even when the system is asymptotically stable, it is still relevant to use a stateobserver in order to accelerate the convergence of the system mass or of the state trajectories to the desired values.

\section{Compartmental Systems}

Compartmental systems are dynamical systems described by a set of equations of the form

$$
\begin{aligned}
& \dot{x}_{i}=\sum_{j \neq i} f_{j i}(x)-\sum_{l \neq i} f_{i l}(x)-f_{i 0}(x)+f_{0 i}(x) \\
& i=1, \ldots, n
\end{aligned}
$$

(see [4] or [12]) where $x=\left(x_{1}, \ldots, x_{n}\right)^{T}$ is the state variable and $x_{i}$ and $f_{i j}$ take nonnegative values. Each equation describes the evolution of the quantity or concentration of material within a subsystem, called compartment. Since the compartments exchange with each other and with the environment, in the above equation, $x_{i}$ is the amount (or concentration) of material in compartment $i$, $f_{i j}$ is the flow rate from compartment $j$ to compartment $i$ and the subscript 0 denotes the environment (see [4]). In this paper, we consider the class of linear time-invariant compartmental systems described by

$$
\dot{x_{i}}=\sum_{j \neq i} k_{j i} x_{j}-\sum_{l \neq i} k_{i l} x_{i}-q_{i} x_{i}+b_{i} u, i=1, \ldots, n,
$$

where $x_{i}$ and the input $u$ take nonnegative values, $k_{i j}, q_{i}, b_{i} \in \mathbb{R}_{+}$and at least one $b_{i}$ is positive (see Fig. 1). Note that, in this case, $f_{j i}=k_{j i} x_{j}, f_{0 i}=b_{i} u$ and $f_{i 0}=q_{i} x_{i}$, and it can be easily proved that the system is positive, this is, if we consider an input $u$ that remains nonnegative, then the state variable also remains 
nonnegative. Moreover, (1) can also be written in matrix form as

$$
\dot{x}=A x+b u,
$$

where $A$ (called compartmental matrix) is so that

$$
a_{i i}=-q_{i}-\sum_{j \neq i} k_{i j} \text { and, if } i \neq j, a_{i j}=k_{j i},
$$

and $b=\left(b_{1}, b_{2}, \ldots, b_{n}\right)^{T}$.

The total mass of the system in a given state $x$ is defined as $M(x)=\sum_{i=1}^{n} x_{i}$. For an arbitrary positive value $M^{*}$, the set $\Omega_{M^{*}}=\left\{x \in \mathbb{R}_{+}^{n}: M(x)=M^{*}\right\}$ of all the points $x$ in the state space with mass $M^{*}$ is called an iso-mass.

An important issue in the context of the control of compartmental systems is to design a control law which yields a positive input that steers the system mass $M(x)$ to a desired value.

In [1], the following positive control law is proposed to guarantee that the trajectories converge to a set $\Omega_{M^{*}}$ :

$$
\begin{aligned}
& u(x)=\max (0, \tilde{u}(x)) \\
& \tilde{u}(x)=\left(\sum_{i=1}^{n} b_{i}\right)^{-1}\left(\sum_{i=1}^{n} q_{i} x_{i}+\lambda\left(M^{*}-M(x)\right)\right),
\end{aligned}
$$

where $\lambda$ is an arbitrary design parameter.

In order to state the corresponding theorem, we need to introduce the following concept of full outflow connectedness. A compartmental system (1) is said to be fully outflow connected if at every state $x$ there is a path $i \rightarrow j \rightarrow k \rightarrow \cdots \rightarrow l$ with positive $k_{i j}$ 's from every compartment $i$ to some compartment $l$ such that $q_{l}>0$ (see [1]).

Theorem 1: [1] Let (2) be a fully outflow connected compartmental system. Then, for the closed loop system (2)-(3) with arbitrary initial conditions $x(0) \in \mathbb{R}_{+}^{n}$ :

$i$ ) the iso-mass $\Omega_{M^{*}}$ is forward invariant;

ii) the state $x(t)$ is bounded for all $t>0$ and converges to the iso-mass $\Omega_{M^{*}}$.

The proof of this result is based on the application of LaSalle's invariance principle (see [7], pg.30), by considering the Lyapunov function

$$
V(x)=\frac{1}{2}\left(M^{*}-M(x)\right)^{2}
$$

of (2) on $\mathbb{R}_{+}^{n}$.

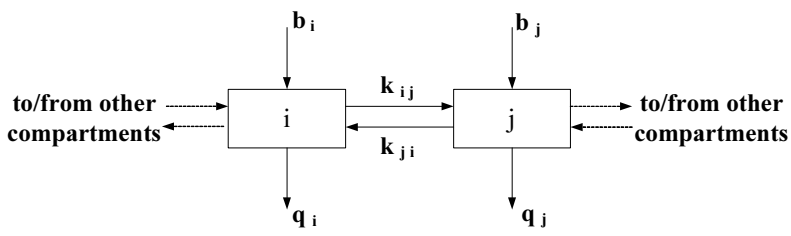

Fig. 1. Two compartments of a linear time-invariant compartmental model, as described by (1).

\section{OBSERVERS}

In most practical cases, the physical state of a system cannot be determined by direct observation. In these cases, we often use a state observer to estimate the state from the knowledge of the input and the output of the real system.

In this section, we analyse the effect of replacing the unknown state of the system by its estimate provided by an observer for control purposes.

Consider the system described by

$$
\left\{\begin{aligned}
\dot{x} & =A x+b u \\
y & =C x
\end{aligned}\right.
$$

where $A$ is a compartmental matrix, $b=$ $\left(b_{1}, b_{2}, \ldots, b_{n}\right)^{T}$ (with $b_{i} \in \mathbb{R}_{+}$and at least one $b_{i}$ positive) and let the closed-loop state estimator $\Omega$ be described by

$$
\left\{\begin{array}{l}
\dot{\hat{x}}=(A-L C) \hat{x}+b u+L y, \\
\hat{y}=\hat{x}
\end{array}\right.
$$

where $\hat{x}$ is the estimated state and $L=\left(l_{1}, l_{2}, \ldots, l_{n}\right)^{T}$

is a vector of gains such that $\sigma(A-L C) \subset \mathbb{C}^{-}$. In order to guarantee the existence of such $L$, we assume that $(C, A)$ is detectable.

If we take the estimated state $\hat{x}$ instead of the real state $x$, the control law to be applied to (4) is:

$$
\begin{aligned}
& u(\hat{x})=\max (0, \tilde{u}(\hat{x})) \\
& \tilde{u}(\hat{x})=\left(\sum_{i=1}^{n} b_{i}\right)^{-1}\left(\sum_{i=1}^{n} q_{i} \hat{x}_{i}+\lambda\left(M^{*}-M(\hat{x})\right)\right) .
\end{aligned}
$$

In the sequel, we analyse the convergence of the state trajectories of the closed loop system (4)-(5).

\section{A. Total mass convergence}

Theorem 2: Let (4) be a compartmental system such that $q_{i} \neq 0, i=1, \ldots, n$. Then, the state trajectories $x(t)$ of the closed loop system (4)-(5), with arbitrary initial conditions $x(0) \in \mathbb{R}_{+}^{n}$, converge to the set

$$
\Omega=\left\{x \in \mathbb{R}_{+}^{n}: M(x)=M^{*}\right\} .
$$

Proof: If we define $e(t)=\hat{x}(t)-x(t)$ as the error between the real and the estimated state, the control law (5) becomes

$$
\begin{aligned}
u(\hat{x}) & =\max (0, \tilde{u}(\hat{x})) \\
\tilde{u}(\hat{x}) & =\left(\sum_{i=1}^{n} b_{i}\right)^{-1}\left(\sum_{i=1}^{n} q_{i}\left(x_{i}+e_{i}\right)+\lambda\left(M^{*}-\right.\right. \\
& \left.\left.-M\left(x_{i}+e_{i}\right)\right)\right) \\
& =\left(\sum_{i=1}^{n} b_{i}\right)^{-1}\left(\sum_{i=1}^{n} q_{i} x_{i}+\lambda\left(M^{*}-+\right.\right. \\
& \left.\left.-M\left[\begin{array}{c}
x \\
e
\end{array}\right]\right)\right) \\
& \left(\sum_{i=1}^{n} b_{i}\right)^{-1} \sum_{i=1}^{n} q_{i} e_{i},
\end{aligned}
$$


Since $L$ is such that $\sigma(A-L C) \subset \mathbb{C}^{-}$,

$$
e(t)=\hat{x}(t)-x(t) \rightarrow 0,
$$

that is, $\hat{x}(t) \rightarrow x(t)$. Therefore, $\forall \varepsilon_{1}>0 \exists t_{1}>0: \forall t>$ $t_{1},-\varepsilon_{1}<e_{i}(t)<\varepsilon_{1}$.

Consider $\varepsilon_{1}>0$ and take $t_{1}>0$ such that the previous condition is verified. Then, since $-\sum_{i=1}^{n} q_{i} \varepsilon_{1}<$ $\sum_{i=1}^{n} q_{i} e_{i}<\sum_{i=1}^{n} q_{i} \varepsilon_{1}$, we have

$$
\begin{aligned}
\tilde{u}(\hat{x}) & >\left(\sum_{i=1}^{n} b_{i}\right)^{-1}\left(\sum_{i=1}^{n} q_{i} x_{i}+\lambda\left(M^{*}-\frac{\sum_{i=1}^{n} q_{i}}{\lambda} \varepsilon_{1}-\right.\right. \\
& \left.\left.-M\left[\begin{array}{l}
x \\
e
\end{array}\right]\right)\right)
\end{aligned}
$$

and

$$
\begin{aligned}
\tilde{u}(\hat{x}) & <\left(\sum_{i=1}^{n} b_{i}\right)^{-1}\left(\sum_{i=1}^{n} q_{i} x_{i}+\lambda\left(M^{*}+\frac{\sum_{i=1}^{n} q_{i}}{\lambda} \varepsilon_{1}-\right.\right. \\
& \left.\left.-M\left[\begin{array}{l}
x \\
e
\end{array}\right]\right)\right) .
\end{aligned}
$$

In the following we take $M_{1}^{*}=M^{*}-\frac{\sum_{i=1}^{n} q_{i}}{\lambda} \varepsilon_{1}, M_{2}^{*}=$ $M^{*}+\frac{\sum_{i=1}^{n} q_{i}}{\lambda} \varepsilon_{1}$ and suppose that $\sum_{i=1}^{n} l_{j} \geq 0$.

In this case, when $\tilde{u}(\hat{x}) \geq 0$,

$$
\begin{aligned}
\dot{M}\left[\begin{array}{l}
x \\
e
\end{array}\right] & =\lambda\left(M^{*}-M\left[\begin{array}{l}
x \\
e
\end{array}\right]\right)-\sum_{i=1}^{n}\left(\sum_{i=1}^{n} l_{j}\right) e_{i} \\
& =\lambda\left(M^{*}-M\left[\begin{array}{l}
x \\
e
\end{array}\right]\right)-\left(\sum_{i=1}^{n} l_{j}\right) M(e) \\
& \leq \lambda\left(M^{*}+\frac{n\left(\sum_{i=1}^{n} l_{j}\right)}{\lambda} \varepsilon_{1}-M\left[\begin{array}{l}
x \\
e
\end{array}\right]\right)
\end{aligned}
$$

and

$$
\begin{aligned}
\dot{M}\left[\begin{array}{l}
x \\
e
\end{array}\right] & =\lambda\left(M^{*}-M\left[\begin{array}{l}
x \\
e
\end{array}\right]\right)-\left(\sum_{i=1}^{n} l_{j}\right) M(e) \\
& \geq \lambda\left(M^{*}-\frac{n\left(\sum_{i=1}^{n} l_{j}\right)}{\lambda} \varepsilon_{1}-M\left[\begin{array}{l}
x \\
e
\end{array}\right]\right) .
\end{aligned}
$$

Define

$$
\begin{aligned}
& \bar{M}_{1}=M^{*}-\frac{n\left(\sum_{i=1}^{n} l_{j}\right)}{n\left(\sum_{i=1}^{\lambda} l_{j}\right)} \varepsilon_{1}, \\
& \lambda \varepsilon_{1}=M^{*}+\frac{n}{\lambda},
\end{aligned}
$$

and let $M_{1}=\min \left\{\bar{M}_{1}, M_{1}^{*}\right\}$ and $M_{2}=\bar{M}_{2}$.

Consider the function $V: \mathbb{R}^{n} \rightarrow \mathbb{R}$ defined by

$$
\begin{aligned}
& V\left[\begin{array}{l}
x \\
e
\end{array}\right]= \\
& = \begin{cases}\frac{1}{2}\left(M\left[\begin{array}{l}
x \\
e
\end{array}\right]-M_{1}\right)^{2} & \text { if } M\left[\begin{array}{l}
x \\
e
\end{array}\right]<M_{1} \\
\frac{1}{2}\left(M\left[\begin{array}{l}
x \\
e
\end{array}\right]-M_{2}\right)^{2} & \text { if } M\left[\begin{array}{l}
x \\
e
\end{array}\right]>M_{2} \\
0 & \text { otherwise. }\end{cases}
\end{aligned}
$$

Note that $V$ is a Lyapunov function of the system in $\mathbb{R}_{+}^{n} \times B\left(0, \varepsilon_{1}\right)$ because it is continuous and $\dot{V}\left[\begin{array}{l}x \\ e\end{array}\right] \leq$
$0, \forall\left[\begin{array}{l}x \\ e\end{array}\right] \in \mathbb{R}_{+}^{n} \times B\left(0, \varepsilon_{1}\right)$ (see [7], pg.30). In fact, if $\left[\begin{array}{l}x \\ e\end{array}\right] \in \mathbb{R}_{+}^{n} \times B\left(0, \varepsilon_{1}\right)$,

$$
\begin{aligned}
& \dot{V}\left[\begin{array}{l}
x \\
e
\end{array}\right]= \\
& = \begin{cases}\left(M\left[\begin{array}{l}
x \\
e
\end{array}\right]-M_{1}\right) \dot{M}\left[\begin{array}{l}
x \\
e
\end{array}\right] & \text { if } M\left[\begin{array}{l}
x \\
e
\end{array}\right]<M_{1} \\
\left(M\left[\begin{array}{l}
x \\
e
\end{array}\right]-M_{2}\right) \dot{M}\left[\begin{array}{l}
x \\
e
\end{array}\right] & \text { if } M\left[\begin{array}{l}
x \\
e
\end{array}\right]>M_{2} \\
0 & \text { otherwise }\end{cases}
\end{aligned}
$$

is nonpositive, as we next show.

- Suppose that $M\left[\begin{array}{l}x \\ e\end{array}\right]<M_{1}$. In this case, $\tilde{u}(\hat{x})$ and $\dot{M}\left[\begin{array}{l}x \\ e\end{array}\right]$ will be positive if $\varepsilon_{1}$ is such that

$$
\left\{\begin{array}{l}
\frac{n\left(\sum_{i=1}^{n} L_{j}\right)}{\lambda} \varepsilon_{1}<M^{*} . \\
\frac{\sum_{i=1}^{n} q_{i}}{\lambda} \varepsilon_{1}<M^{*}
\end{array} .\right.
$$

Since $\varepsilon_{1}>0$ is arbitrary, we shall suppose that this is the case. Note that, in this case,

$$
\dot{V}\left[\begin{array}{l}
x \\
e
\end{array}\right]=\left(M\left[\begin{array}{l}
x \\
e
\end{array}\right]-M_{1}\right) \dot{M}\left[\begin{array}{l}
x \\
e
\end{array}\right]<0 .
$$

- Suppose that $M\left[\begin{array}{l}x \\ e\end{array}\right]>M_{2}$. In this case, if $\tilde{u}(\hat{x}) \geq$ $0, \dot{M}\left[\begin{array}{l}x \\ e\end{array}\right]<0$. If $\tilde{u}(\hat{x})<0, u(\hat{x})=0$ and

$$
\begin{aligned}
& \dot{M}\left[\begin{array}{l}
x \\
e
\end{array}\right]= \\
& =-\sum_{i=1}^{n} q_{i} x_{i}-\sum_{i=1}^{n} q_{i} e_{i}-\left(\sum_{i=1}^{n} l_{j}\right) M(e) \\
& \leq-q_{\min } M(x)+\sum_{i=1}^{n} q_{i} \varepsilon_{1}+n\left(\sum_{i=1}^{n} l_{j}\right) \varepsilon_{1} \\
& <-q_{\min } M_{2}+q_{\min } M(e)+\sum_{i=1}^{n} q_{i} \varepsilon_{1}+ \\
& +n\left(\sum_{i=1}^{n} l_{j}\right) \varepsilon_{1} \\
& <-q_{\min } M_{2}+\left(n q_{\min }+\sum_{i=1}^{n} q_{i}+n\left(\sum_{i=1}^{n} l_{j}\right)\right) \varepsilon_{1} .
\end{aligned}
$$

Since $q_{\min } M_{2}>0, \quad$ it is possible to choose $\varepsilon_{1}>0$ such that $-q_{\min } M_{2}+$ $\left(n q_{\text {min }}+\sum_{i=1}^{n} q_{i}+\sum_{i=1}^{n}\left(\sum_{i=1}^{n} l_{j}\right)\right) \varepsilon_{1}<0$. Then, for $t \geq t_{1}, \dot{M}\left[\begin{array}{l}x \\ e\end{array}\right]<0$ and

$$
\dot{V}\left[\begin{array}{l}
x \\
e
\end{array}\right]=\left(M\left[\begin{array}{l}
x \\
e
\end{array}\right]-M_{2}\right) \dot{M}\left[\begin{array}{l}
x \\
e
\end{array}\right]<0 \text {. }
$$

Applying LaSalle's invariance principle (see [7], pg.30), it turns out that $\left[\begin{array}{l}x \\ e\end{array}\right](t)$ converges to the largest invariant set contained in 


$$
\begin{aligned}
& \left\{\left[\begin{array}{l}
x \\
e
\end{array}\right] \in \mathbb{R}_{+}^{n} \times B\left(0, \varepsilon_{1}\right): \dot{V}\left[\begin{array}{l}
x \\
e
\end{array}\right]=0\right\}= \\
& =\left\{\left[\begin{array}{l}
x \\
e
\end{array}\right] \in \mathbb{R}_{+}^{n} \times B\left(0, \varepsilon_{1}\right): M\left[\begin{array}{l}
x \\
e
\end{array}\right] \in\left[M_{1}, M_{2}\right]\right\} .
\end{aligned}
$$

Then, $M\left[\begin{array}{l}x \\ e\end{array}\right] \rightarrow\left[M_{1}, M_{2}\right]$.

The case where $\sum_{i=1}^{n} l_{j}<0$ is similar to the previous one.

Note that the definitions of $M_{1}$ and $M_{2}$ allow to conclude that there is a neighbourhood of $M^{*}, \mathcal{N}=$ ]$M^{*}-\varepsilon, M^{*}+\varepsilon\left[\right.$, with $\varepsilon>0$, such that $\mathcal{N} \supset\left[M_{1}, M_{2}\right]$. Indeed, if $\varepsilon=\max \left\{\frac{n\left|\sum_{i=1}^{n} l_{j}\right|}{\lambda} \varepsilon_{1}, \frac{\sum_{i=1}^{n} q_{i}}{\lambda} \varepsilon_{1}\right\}$, it is easy to verify the previous inclusion. Since $\varepsilon_{1}$ can be taken as small as we want, we prove that $M\left[\begin{array}{l}x \\ e\end{array}\right] \rightarrow M^{*}$. Thus, as $M(e) \rightarrow 0$, we prove that $M(x) \stackrel{\rightarrow}{\rightarrow} M^{*}$ as desired.

Remark 3: The construction of positive observers is an important issue in the theory of linear positive systems. However here the observer system is not required to be positive, since the control law $u$ is subject to a positivity constraint. Note moreover that this positivity constraint is necessary even when the (positive) real state $x$ is used instead of the estimate $\hat{x}$.

\section{B. The neuromuscular blockade case}

In [10], the control law (3) was applied for the control of the neuromuscular blockade of patients undergoing surgery, by means of the infusion of atracurium. In fact, it is possible to model this problem as a threecompartmental model that can be described as depicted in Fig. 2, where $u$ is the drug infusion dose administered in the central compartment, and $k_{12}, k_{21}, k_{13}, q_{3}$ are positive micro-rate constants and $q_{1}, q_{2}$ are nonnegative micro-rate constants that vary from patient to patient. In this case, the set of equations (1) becomes

$$
\left\{\begin{array}{l}
\dot{x}_{1}=-\left(k_{12}+k_{13}+q_{1}\right) x_{1}+k_{21} x_{2}+u \\
\dot{x}_{2}=k_{12} x_{1}-\left(k_{21}+q_{2}\right) x_{2} \\
\dot{x}_{3}=k_{13} x_{1}-q_{3} x_{3}
\end{array}\right.
$$

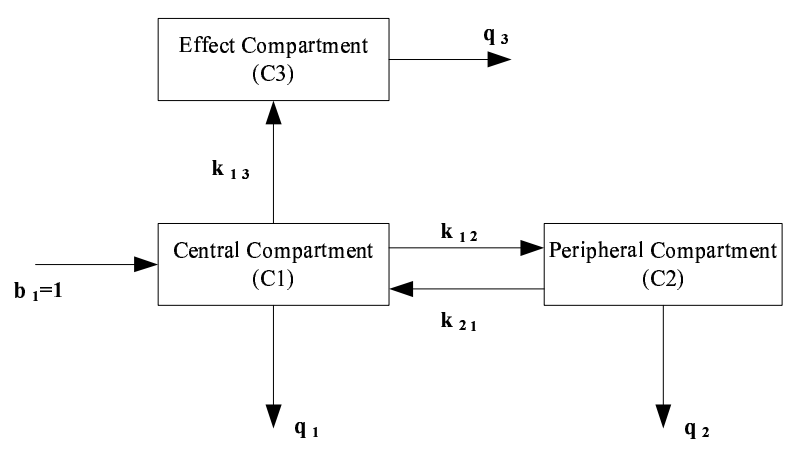

Fig. 2. Compartmental model for the neuromuscular blockade effect of the drug atracurium. where $x_{1}, x_{2}$ and $x_{3}$ are the drug amounts in the central, peripheral and effect compartments, respectively.

It was proved in [10] and in [13], that the application of the control law (3) to a system of the form (8) not only leads the mass to a certain value $M^{*}$, but also leads the whole system state to an equilibrium point $x^{M^{*}}$. Here, we shall prove that a similar result still holds when we control our system (8), using a control law (5).

Although we focus on the administration of a muscular relaxant, our results are also valid for other compartmental systems with the same structure.

The next proposition is useful in the proof of the main result of this subsection.

Proposition 4: Suppose that $q_{i} \neq 0, i=1, \ldots, n$. Then, when the control law (5) is applied to (4), there exists an instant $t_{1}>0$ such that, for $t \geq t_{1}$,

$$
u(\hat{x}(t))=\widetilde{u}(\hat{x}(t)) \geq 0
$$

Proof: According to the previous Theorem, when the control law (5) is applied to (4), the system mass converges to $M^{*}$. This implies that, for every $\varepsilon>0$, there exists an instant $t_{1}>0$ such that

$$
M(x) \in\left[M^{*}-\varepsilon, M^{*}+\varepsilon\right],
$$

for $t \geq t_{1}$. Since $e(t) \rightarrow 0$, there is also a positive instant such that $-\varepsilon<e_{i}<\varepsilon, i=1, \ldots, n$, from that instant on.

Suppose that $t_{1}>0$ is such that the two previous conditions are verified for $t \geq t_{1}$. Thus, for $t \geq t_{1}$,

$$
\begin{aligned}
& \tilde{u}(\hat{x})= \\
& =\left(\sum_{i=1}^{n} b_{i}\right)^{-1}\left(\sum_{i=1}^{n} q_{i} x_{i}+\sum_{i=1}^{n} q_{i} e_{i}+\right. \\
& \left.+\lambda\left(M^{*}-M(x)-M(e)\right)\right) \\
& \geq\left(\sum_{i=1}^{n} b_{i}\right)^{-1}\left(q_{\min } M(x)-\sum_{i=1}^{n} q_{i} \varepsilon+\right. \\
& \left.+\lambda M^{*}-\lambda\left(M^{*}+\varepsilon\right)-\lambda n \varepsilon\right) \\
& \geq\left(\sum_{i=1}^{n} b_{i}\right)^{-1}\left(q_{\min }\left(M^{*}-\varepsilon\right)-\sum_{i=1}^{n} q_{i} \varepsilon+\right. \\
& \left.+\lambda M^{*}-\lambda\left(M^{*}+\varepsilon\right)-\lambda n \varepsilon\right) \\
& =\left(\sum_{i=1}^{n} b_{i}\right)^{-1}\left[q_{\min } M^{*}-\left(q_{\min }+\sum_{i=1}^{n} q_{i}+\lambda(n+1)\right) \varepsilon\right]
\end{aligned}
$$

and, if

$$
\varepsilon \leq \frac{q_{\min }}{q_{\min }+\sum_{i=1}^{n} q_{i}+\lambda(n+1)} M^{*},
$$

it is easily seen that $u(\hat{x}(t))=\widetilde{u}(\hat{x}(t)) \geq 0$.

It was shown in[10] and in [13] that the application of the control law (3) to a system of the form (8) leads the whole system state to the unique steady state $x^{M^{*}}$ with mass $M^{*}, x^{M^{*}}=\left[\begin{array}{lll}\alpha_{1} & \alpha_{2} & 1\end{array}\right]^{T} \frac{M^{*}}{\alpha_{1}+\alpha_{2}+1}$, where $\alpha_{1}$ and $\alpha_{2}$ depend on the micro-rate constants $k_{i j}$ and $q_{i}$. In the following, we shall prove that this result holds when we consider the control law (5) instead. 
Proposition 5: Consider that $q_{i} \neq 0, i=1,2,3$. The state trajectories $x(t)$ of the closed loop system (8)-(5), with arbitrary initial conditions $x(0) \in \mathbb{R}_{+}^{n}$, converge to $x^{M^{*}}$.

Proof: Define $F=\left[\begin{array}{lll}q_{1}-\lambda & q_{2}-\lambda & q_{3}-\lambda\end{array}\right]$ and $v=\lambda M^{*}$.

According to Proposition 4 , there exists an instant $t_{1}>$ 0 such that, for $t \geq t_{1}, u(\hat{x}(t))=\widetilde{u}(\hat{x}(t)) \geq 0$. Thus, for $t \geq t_{1}$, we obtain the following closed loop system:

$$
\left\{\begin{array}{rl}
{\left[\begin{array}{l}
\dot{x} \\
e
\end{array}\right]} & =\left[\begin{array}{cc}
A+b F & b F \\
0 & A-L C
\end{array}\right]\left[\begin{array}{l}
x \\
e
\end{array}\right]+\left[\begin{array}{l}
b \\
0
\end{array}\right] v \\
y & =\left[\begin{array}{ll}
C & 0
\end{array}\right]\left[\begin{array}{l}
x \\
e
\end{array}\right]
\end{array} .\right.
$$

Let

$$
A_{1}=\left[\begin{array}{cc}
A+b F & b F \\
0 & A-L C
\end{array}\right] .
$$

Since it can be easily seen (using the Routh - Hurwitz stability criterion) that, all the eigenvalues of $A+b F$ lie in $\mathbb{C}^{-}$, it turns out that $A_{1}$ is asymptotically stable. Thus,

$$
\begin{aligned}
{\left[\begin{array}{l}
x \\
e
\end{array}\right](t) } & =e^{A_{1} t}\left[\begin{array}{l}
x \\
e
\end{array}\right](0)+\int_{0}^{t} e^{A_{1}(t-\tau)}\left[\begin{array}{l}
b \\
0
\end{array}\right] v d \tau \\
& =e^{A_{1} t}\left[\begin{array}{l}
x \\
e
\end{array}\right](0)+\int_{0}^{t} e^{A_{1}(t-\tau)} d \tau\left[\begin{array}{l}
b \\
0
\end{array}\right] v \\
& =e^{A_{1} t}\left[\begin{array}{l}
x \\
e
\end{array}\right](0)+A_{1}^{-1}\left(e^{A_{1} t}-I\right)\left[\begin{array}{l}
b \\
0
\end{array}\right] v
\end{aligned}
$$

and, since $e^{A_{1} t} \rightarrow 0$, it turns out that $\left[\begin{array}{l}x \\ e\end{array}\right](t) \rightarrow$ $-A_{1}^{-1}\left[\begin{array}{l}b \\ 0\end{array}\right] v$.

However,

$$
\begin{aligned}
& A_{1}^{-1}=\left[\begin{array}{cc}
A+b F & b F \\
0 & A-L C
\end{array}\right]^{-1}= \\
& =\left[\begin{array}{cc}
(A+b F)^{-1} & -(A+b F)^{-1} b F(A-L C)^{-1} \\
0 & (A-L C)^{-1}
\end{array}\right]
\end{aligned}
$$

Therefore,

$$
-A_{1}^{-1}\left[\begin{array}{l}
b \\
0
\end{array}\right] v=-\left[\begin{array}{c}
(A+b F)^{-1} b v \\
0
\end{array}\right]
$$

and it turns out that

$$
x(t) \rightarrow-(A+b F)^{-1} b v .
$$

Since it is easy to verify that $-(A+b F)^{-1} b v$ coincides with $x^{M^{*}}$, this proves our result.

1) Simulations: In the sequel, some simulation examples are presented for the control of the administration of the neuromuscular relaxant drug atracurium to patients undergoing surgery. As it was already shown, it is possible to model this problem as a three compartmental model described by the set of equations (8).

Here, we consider that the patient's real model is given by (8), with the following values for the parameters $\left(\right.$ units $\left.=\min ^{-1}\right): k_{12}=0.2131, k_{13}=0.0017, k_{21}=$ $0.1252, q_{1}=0.1047, q_{2}=0.01, q_{3}=0.0836$.

Our aim is to stabilize the system mass on the value $M^{*}=143.5997$ and the third component of the system state (which corresponds to the drug effect) on $x_{3}^{M^{*}}=$ 1.1169 (which, in an exact modeling situation, can be shown to correspond to the typical $10 \%$ level of neuromuscular blockade), applying the control law (5) to (8).

The following simulations illustrate the behavior of the mass and of the drug effect for the controlled system for different observer gain vectors $L$. In the first and in the second simulations, depicted in Fig. 3, it is assumed that $L=0$. Note that this corresponds to a parallel computation of the state based on initial conditions that are affected by error. However, since the system turns out to be asymptotically stable, this initial error will converge to zero. The other simulations show how the convergence can be accelerated by the choice of suitable observer gains.

In accordance with our theoretical results, in all simulations one observes that the system mass converges to $M^{*}$ and the drug effect converges to $x_{3}^{M^{*}}$.

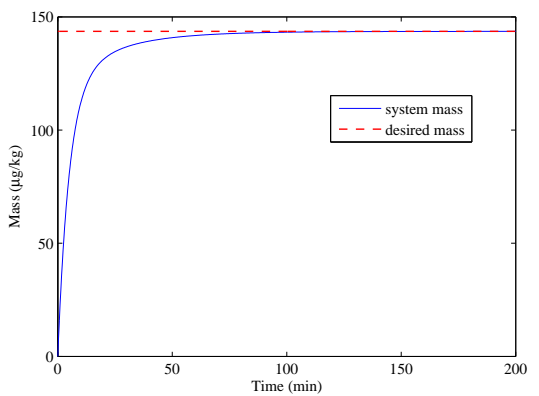

(a)

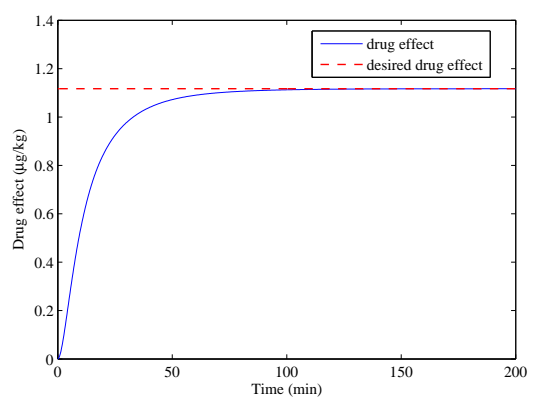

(b)

Fig. 3. Simulations for the neuromuscular blockade case for $L=0$. Observer poles: $-0.0836,-0.0398$ and -0.4149 . (a) Simulation for the control of the system mass. Convergence time: $149.7 \mathrm{~min}$. (b) Simulation for the control of the drug effect. Convergence time: $156.3 \mathrm{~min}$. 


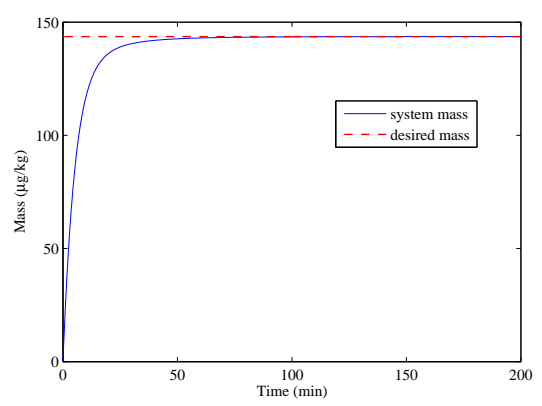

(a)

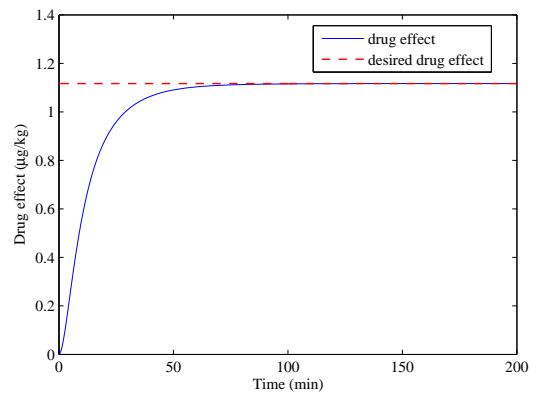

(b)

Fig. 4. Simulations for the neuromuscular blockade case for $L=\left[\begin{array}{lll}-0.9521 & 0.7880 & 0.0127\end{array}\right]^{T}$. Observer poles: $-0.0905,-0.0405$ and -0.42 . (a) Simulation for the control of the system mass. Convergence time: $118.3 \mathrm{~min}$. (b) Simulation for the control of the drug effect. Convergence time: $127 \mathrm{~min}$.

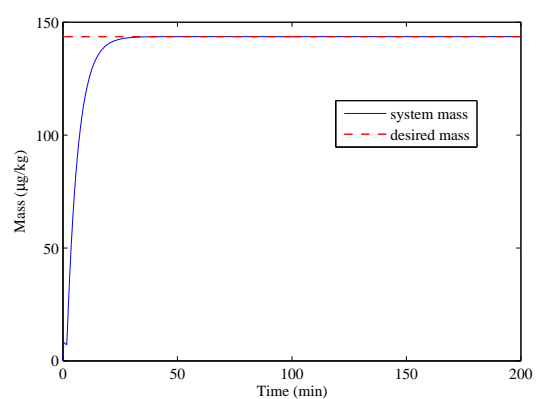

(a)

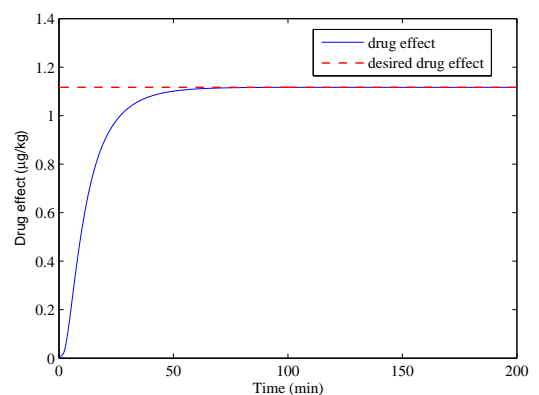

(b)

Fig. 5. Simulations for the neuromuscular blockade case for $L=\left[\begin{array}{lll}0.1686 & 4.5141 & 0\end{array}\right]^{T}$. Observer poles: -10 with multiplicity 3. (a) Simulation for the control of the system mass. Convergence time: $39.67 \mathrm{~min}$. (b) Simulation for the control of the drug effect. Convergence time: $92.33 \mathrm{~min}$.

\section{CONCLUSION}

This paper presents an analysis of the performance of state-observers in the control of compartmental systems under the presence of uncertainties in the system initial state.

It turns out that, in this case, the asymptotic mass values coincide with those obtained when the state of the real system is supposed to be known. Moreover, we prove that, for a class of three-compartmental systems of interest, the steady state of the controlled system is the same as in the exact case. Our results are illustrated by several simulations, which show the role of observers in the acceleration of the convergence.

\section{ACKNOWLEDGMENT}

This work was partially supported by FCT through the Unidade de Investigação Matemática e Aplicações (UIMA), Universidade de Aveiro, Portugal.

\section{REFERENCES}

[1] Bastin, G. and A. Provost (2002). "Feedback stabilisation with positive control of dissipative compartmental systems". In: Proceedings of the 15th International Symposium on Mathematical Theory of Networks and Systems. Notre Dame, Indiana, USA.

[2] Luenberger, D. G. (1964). "Observing the state of a linear system", IEEE Transactions on Military Electronics, vol. 23, pp. 119-125.

[3] Luenberger, D. G. (1966). "An introduction to observers", IEEE Transactions on Automatic Control, vol. 16, pp. 596-602.

[4] Godfrey, K. (1983). Compartmental Models and Their Application. Academic Press.

[5] Haddad, W., T. Hayakawa and J. Bayley (2003). " Adaptive control for non-negative and compartmental dynamical systems with applications to general anesthesia". International Journal of Adaptive Control and Signal Processing, vol. 17, pp. 209-235.

[6] Jacquez, J. and C. Simon (1993). "Qualitative theory of compartmental systems". SIAM Review, vol. 35, no. 1, pp. 43-79.

[7] LaSalle, J. P. (1976). The Stability of Dynamical Systems. SIAM, Bristol, England.

[8] Lemos, J., T. Mendonça and E. Mosca (1991). "Long-range adaptive control with input constraints". International Journal of Control, vol. 54, pp. 289-306.

[9] Linkens, D. (1994). Intelligent control in biomedicine. Taylor and Francis, London.

[10] Magalhães, H. (2005). "Identification and control of positive and compartmental systems applied to neuromuscular blockade". In: Preprints of the 16th IFAC World Congress. Prague, Czech Republic.

[11] Mendonça, T. and P. Lago (1998). "PID control strategies for the automatic control of neuromuscular blockade". Control Engineering Practice, vol. 6, pp. 1225-1231.

[12] Sandberg, W. (1978). "On the mathematical foundations of compartmental analysis in biology, medicine and ecology", IEEE Transactions on Circuits and Systems, vol. 25, pp. 273-279.

[13] Sousa, C., T. Mendonça and P. Rocha (2008). "Total mass control in uncertain compartmental systems". In: Proceedings of the 8th Portuguese Conference on Automatic Control. Vila Real, Portugal. 